\title{
A Survey of Game Theory Applications in Electrical Power Micro-Grid Systems
}

\author{
Osama Abdel-Raouf \\ Operations Research and \\ Decision Support Department \\ Faculty of Computers and \\ Information, Menoufia University, \\ Menoufia, Egypt
}

\author{
M. A. Elsisy \\ Basic Engineering Sciences \\ Department, \\ Benha Faculty of Engineering, \\ Benha University, \\ Benha, Egypt
}

\author{
E. F. Kelash \\ Basic Engineering Sciences \\ Department, \\ Benha Faculty of Engineering, \\ Benha University, \\ Benha, Egypt
}

\begin{abstract}
The technology of Smart Grid is believed to be the future of power system networks. Smart Grid (SG) gains its importance due to its proven ability to improve stability, efficiency and robustness of electrical power grids. SG system consists mainly of two components which are electrical distribution system and communication layer. In the electrical distribution system, the generated energy comes from a network of distributed energy resources "DERs", which is called microgrid. In most cases, these DERs are recommended to be renewable energy sources "RESs" to reduce emissions and harmful environmental effects. One of the main drawbacks of renewable energy sources is that their availability varies with time and so that the micro-grid technology faces various technical challenges which motivate many researchers to adopt techniques to overcome these challenges. In this regard and due to its capability of studying complex interactions between independent rational players, game theory is expected to have a great contribution in the phase of design and analysis of micro-grids. In this paper, the fundamental concepts of game theory are streamlined, an overview on the applications of game theoretical concepts in various microgrid optimization problems are presented, a novel classification of research points covered by researchers are provided. Finally, some future opportunities that are expected to solve some of the technical challenges facing micro-grid technology are introduced.
\end{abstract}

\section{General Terms}

Game Theory, Micro Grid, Smart Grid.

\section{Keywords}

Game Theory, Cooperative Game Theory, Non-Cooperative Game Theory, Smart Grid, Micro Grid, Distributed Generators.

\section{INTRODUCTION}

In contrast to the recent power system in which electrical generators are constructed in remote geographical areas and power is transmitted at high voltage level, the "micro-grid" $(\mu \mathrm{G})$ is presented as a localized group of electrically connected distributed energy resources (DERs) based mainly on renewable energy sources such as sun and wind at medium and low voltage levels [1]. In general, an $\mu \mathrm{G}$ has two modes of operation, it can operate in "Connected Mode" at which the micro-grid is connected from one side to the macro-grid at medium voltage level and from the other side to the end users (consumers) at low voltage level; the $\mu \mathrm{G}$ can also disconnect from the main utility grid and operate autonomously depending on the power generated from its DERs, this mode is called "Islanded Mode" [2]. The micro-grid system consists of some basic components which have a vital rule in meeting the previously mentioned functionality of the $\mu \mathrm{G}$ in its two modes of operation. Since the main concept of the $\mu \mathrm{G}$ is transforming the distribution grids from passive to active networks [3], then the first component of the $\mu \mathrm{G}$ is a combination of distributed energy generators (DERs) such as photovoltaic arrays, solar cells, micro turbines, fuel cells, wind turbines, etc, together with a set of distributed storage devices (DSs) such as fly wheels, batteries and energy storage capacitors. The second component is a network of medium and low voltage electrical transmission lines which used to connect the DERs, the DSs with the loads. The loads of the micro-grid are the end users "consumers" of electrical power like lighting, refrigerators, micro wave ovens, air conditioners, etc of residential loads beside the commercial and industrial loads. These loads consume an amount of power depending on the need and usage of the user, then they must be controllable and this is because the power generated by the DERs are also variable at different points in the day depending on the weather which has a great effect on the generated amount of power from the renewable energy sources like the sun and the wind. Point of common coupling (PCC) is another main component which used in the vast majority of micro-grids. PCC is the point in where a microgrid is connected to the utility main grid; it is defined to be the electrical component (switch) which is responsible for the selection of the mode of operation of the $\mu \mathrm{G}$. If the PCC is connected, then the $\mu \mathrm{G}$ is operating in connected mode and if it is disconnected, then the $\mu \mathrm{G}$ is operating in islanded mode. In some cases, the interconnection with the main grid is not recommended due to technical or economic issues; in this case, the micro-grid can operate without the PCC and is called isolated micro-grid [4]. Figure 1 shows a simple representation of a micro-grid system. Although $\mu \mathrm{G}$ technology has great advantages over the traditional grid like energy saving through reducing power losses in transmission lines due to the small distance in between micro-grids, reduction of carbon emissions due to the utilization of clean energy resources and decreasing the dependability on the main grid, this technology still facing numerous challenges in various levels such as design, control, analysis and protection against electrical faults [5]. In this respect, Game Theory has been adopted by a number of researchers to solve a lot of optimization problems in micro-grid systems. Game Theory is believed to be one of the promising analytical tools in designing and optimizing not only $\mu \mathrm{G}$ systems, but also other systems that depends in their work on networks such as communication systems and traffic networks. This is because of its capability of analyzing situations at which cooperation 
and/or conflict between participants can occur. The rest of the paper is organized as follows. The basics of Game Theory are discussed in section 2. In section 3, the contributions of Game Theory in resolving some optimization problems in the microgrid system are overviewed in addition to proposing some future opportunities which could be used as research points. Section 4 presents the conclusions of the work.

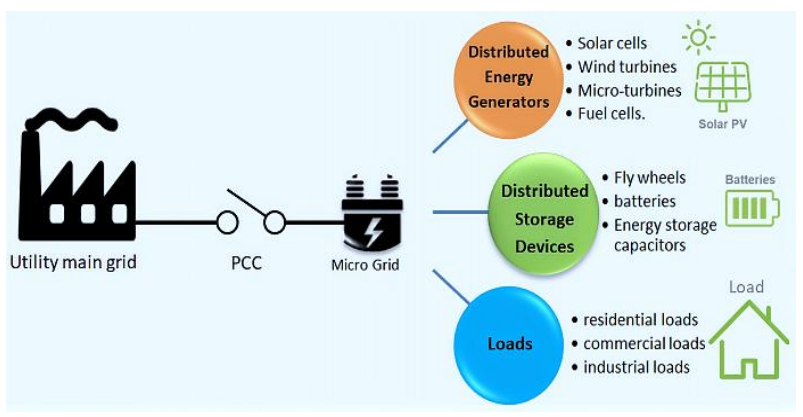

Fig 1: Micro Grid system components.

\section{FUNDAMENTAL CONCEPTS OF GAME THEORY}

In this section, attention is focused on the basic concepts of game theory. Game theory is the branch of applied mathematics which is dedicated to study and analyze the interactions between rational independent decision makers called players [6]. Game theory was firstly developed and presented by the mathematicians John Von Neuman and Oskar Morgenstern in 1944 in their text Theory of Games and Economics Behavior in which they established and defined the rules, components and outcomes of a game [7]. Nash's contribution in non-cooperative games is considered as a corner stone in the field of game theory. Game theory has two main categories cooperative and non-cooperative. In 1950, Nash introduced the equilibrium concept named for him as a stable strategic solution for non-cooperative games in [8] and [9]. In the last few years, game theory was used in many fields like economics, engineering, politics and philosophy [10] which makes it a very powerful tool in modeling and analyzing very complex situations in various disciplines.

In the field of game theory and according to the behavior of players, one can differentiate between three main classes of games: Non-cooperative [8, 11], cooperative games [12-14] and evolutionary game theory which depends on the concepts of Biology [15-17]. From the perspective of game properties, game theory can be categorized according to the strategies used in the game to (pure strategy and mixed strategy games), according to the number of players involved in the game to (two players and n-players game), according to the timing of action to (simultaneous move and sequential move games), and according to the number of game stages to (static and dynamic games), and so on [18]. The taxonomy of games in game theory is shown in figure (2). In the rest of this section, focus is restricted to the classification of games according to the behavior of players and especially to the two main categories "Non-cooperative and cooperative games".

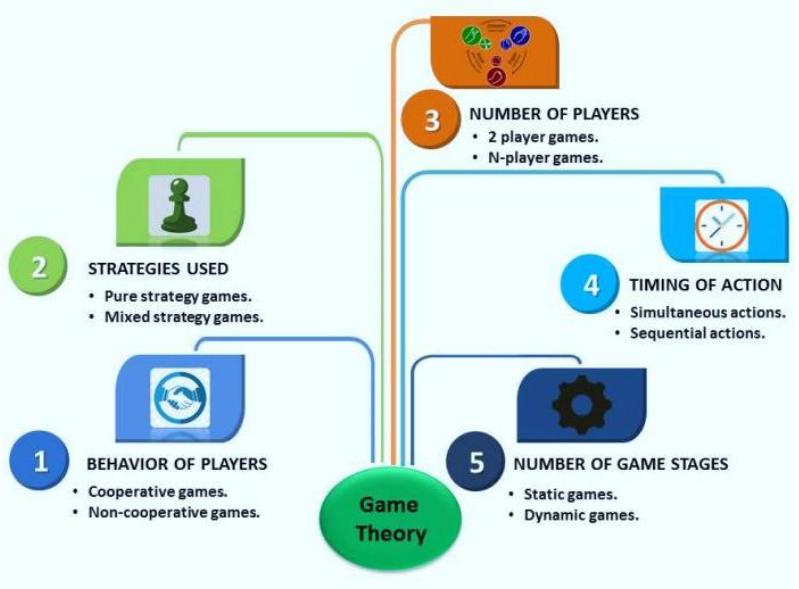

Fig 2: Taxonomy of Game Theory.

\subsection{Non-cooperative game theory}

This branch of game theory is dedicated to study the competitive behavior of decision makers. It deals with the situations at which each player is interested in acting independently to maximize his gain without any collaboration or coordination with any of the other entrants in the game. In this area of game theory, the aim is to study the effect of the lack of cooperation among players that have adverse interests on a certain strategic decision making situation and its effect on the utility achieved by each of them. Non-cooperative games can be classified according to the number of stages of the game to static and dynamic games. Static game is considered to be a simultaneous game as no player has any information about other players' actions. In consequence, the notion of time has no effect on the choices or the decisions of each player. One-off sealed bid auction is an example of static games. In this game, each player has a bid, and no player knows any information about other players' bids and at the end of the game, the player which bid is the greatest is announced to be the winner of the game and the final price equals its bid [19]. In contrast, in dynamic games, players know some information about their competitors' strategies and actions so they can act more than once and so that the time has a vital rule in the process of making decision [11]. Dynamic auction is an example of dynamic games. Here bidders can make more than one bid up the price of a certain object and the bid with the greatest value is the winner of the auction. In the rest of this subsection, the normal form general definition and the solution concepts of non-cooperative games are reviewed.

\section{Definition 1}

A static non-cooperative game can be represented mathematically by the 3 -tuple $\left(\mathcal{N},\left(\mathcal{A}_{i}\right)_{i \in \mathcal{N}},\left(u_{i}\right)_{i \in \mathcal{N}}\right)$ where $\mathcal{N}$ is a finite set of players, $\left(\mathcal{A}_{i}\right)_{i \in \mathcal{N}}$ is the set of strategies that can be selected by players and $\left(u_{i}\right)_{i \in \mathcal{N}}$ is the set of utility functions. Each player $i$ in such a game selects a certain strategy $a_{i}$ from $\mathcal{A}_{i}$ in order to maximize his gains or minimize his losses. This optimization process doesn't depend solely on the action $a_{i}$ selected by the player, but also on the set $a_{-i}$ which denotes the set of strategies selected by all other players $\mathcal{N}-\{i\}$.

In dynamic non-cooperative games, one must take in account the notion of time, the sets of information and the sets of 
former actions that can affect the utility functions of the players. (See [11] for more details).

Solution concepts: As mentioned before, Non-cooperative game theory's main objective is to study the competitive behavior by proposing proper recipes and algorithms. These algorithms should have the ability to analyze such situations and to calculate not only the total outcome of the game but also the pay-off of each player. The most significant solution concept in non-cooperative game theory is the Nashequilibrium. It highlights a situation at which no player can increase his benefits by changing separately his strategy while leaving other players' strategies fixed. The pure strategy Nashequilibrium could be defined as follows.

\section{Definition 2}

The vector $a^{*} \in\left(\mathcal{A}_{i} \times \mathcal{A}_{i}\right)$ is said to be a Nashequilibrium if:

$$
u_{i}\left(a_{i}^{*}, a_{\mathcal{N}-\{i\}}^{*}\right) \geq u_{i}\left(a_{i}, a_{\mathcal{N}-\{i\}}^{*}\right) \forall a_{i} \in \mathcal{A}_{i}
$$

Besides its advantage of characterizing a stable state, Nashequilibrium still has some drawbacks. For example, a game can have multiple equilibriums and it will be difficult to select the more efficient one, also the Nash equilibrium may not exist in pure strategy games i.e. (the existence of it is only guaranteed in mixed strategy games).

Depending on the situation to be analyzed, the type of equilibrium to be used is selected. This is because the existence of several equilibrium definitions such as pure Nash-equilibrium, mixed Nash-equilibrium, perfect equilibrium, correlated equilibrium, strong equilibrium ...etc. Thus one should be aware of the conditions of the game in order to select the appropriate concept for analyzing it.

In micro grid systems, non-cooperative game theory concepts have a lot of applications. Nash equilibrium could be used to determine the optimal contract price of electric power among DG units as in [27] as a solution concept for a bi-level game similar to Stackelberg game. In [31] the Nash equilibrium is used to model the energy market in the distribution level. The authors of [32] use the concepts of non-cooperative games in enhancing the power quality of the electric network by controlling the voltage level during the process of injecting reactive power. Nash equilibrium is also used in [34] to locate and size the DG units in the distribution network in order to reduce the overall power losses of the system.

\subsection{Cooperative game theory}

In contrast to Non-cooperative game theory, players in cooperative games have the ability to coordinate with each other in order to increase their profit from the game by constructing alliances among themselves. Cooperative game theory studies the conditions under which coalitions are constructed, the amount of utility obtained by each coalition and how the total profit is apportioned between the players. Cooperative game theory has applications in various fields such as politics, economics and engineering networks at which the study of alliances constructions is a vital point. The following is the formal definition of cooperative games in the characteristic form.

\section{Definition 3}

A cooperative game in the characteristic form is represented by $(\mathcal{N}, \mathcal{V})$ where $\mathcal{N}$ is a finite set of players and $\mathcal{V}: 2^{\mathcal{N}} \rightarrow \mathfrak{R}$ with $\mathcal{V}(\phi)=0$ is the characteristic function that appoints to each coalition $S \subseteq \mathcal{N}$ a value representing it's worth [14].
Cooperative game theory consists of two main branches: coalitional games and bargaining games.

\subsubsection{Coalitional games}

Coalitional game theory studies the formation of coalitions (cooperative groups) that aim to increase the benefits of players of a certain game. The authors of [6] classified the coalitional games to:

\section{- Canonical coalitional games}

In canonical coalitional games, the more players included in the coalition, the more the utility gained by this coalition. In consequence, the optimal structure of the game is the grand coalition that includes all the game participants in a single coalition. In such a game, the main objective is to study the stability conditions of the grand coalition in addition to the allocation of the payoff in a way that guarantees that no player has the intention to leave the grand coalition to increase its payoff.

\section{- Coalition formation games}

Unlike canonical coalitional games, the formation of a grand coalition is not guaranteed in coalition formation games. The cost of coalition formation here limits the profits gained by each coalition. In this case, the set of players $\mathcal{N}$ is partitioned into a set of coalitions $S$ such that $\bigcup_{i \in N} S_{i}=\mathcal{N}, S_{i} \cap S_{j}=$ $\phi,|S|>1$.

\section{- Coalitional graph games}

This type of coalitional games is used to analyze the games at which the utility of a coalition depends on the topology of the game and the interconnections between the players within the coalition.

The most common solution concepts used for studying coalitional games are: The fair division, the core, the nucleus and the Shapley value. These concepts are used to divide the total worth among the players in a way to guarantee the stability of the game and to assure that no player can improve its payoff by leaving its coalition. (Interested readers are referred to $[12,13,14])$.

\subsubsection{Bargaining games}

Bargaining games study the agreements on the terms under which the players cooperate and the negotiations done between the participants to form the cooperative groups. The most important concept used in bargaining games is the Nash bargaining which shows that the Pareto optimality, linear invariance, symmetry and independence of irrelevant alternatives axioms imply a unique bargaining solution.

The bargaining game is considered to be a 2-person game. Therefore, for a 2-player bargaining game with a set of alternatives $A$, the outcome of the game is either an alternative a s.t $a \in A$ if both of the two bargainers agreed on $a$ or the outcome is $d$ where $d$ denotes the outcome of disagreement if both of them fail to agree on a certain alternative. Assume that the preferences of players 1 and 2 are represented by utility functions $u_{1}$ and $u_{2}$ respectively, then: the set of feasible utility payoffs in the case of agreement is denoted by $S$ where $S=\left\{x: x=\left(u_{1}(a), u_{2}(a)\right)\right.$ and $\left.a \in A\right\}$ and the payoffs of the players in the case of disagreement is $d=\left(u_{1}(d), u_{2}(d)\right)$. The point at which there is no agreement is called the threat point or the disagreement point. The values of $u_{1}, u_{2}$ and $d$ are all the information needed to solve the bargaining problem. The payoff to the bargaining game players can be calculated using the function $\varphi$ s.t $\varphi:(S, d) \rightarrow R$ where $\varphi$ is the function that assigns for every player a real value representing his payoff depending on the values of the utility 
payoffs in the case of agreement $S$ and the payoffs of the players in the case of disagreement $d$. The bargaining game has many solutions such as Nash-bargaining, Kalay and Smorodinsky, and Felsenthal and Diskin solutions. For Nash solution to the bargaining game, the set $S$ should be convex, compact and the existence of $a$ such that $a \in S$ and $a>d$ for both players should be guaranteed. The negation process in this case aims to maximize $\left(a_{1}-d_{1}\right)\left(a_{2}-d_{2}\right)$ s. $t a_{i}$ is the negotiated payoff of player $i$ and $d_{i}$ is the disagreement threat point payoff for player $i$ and $i \in\{1,2\}$. (See [20, 21, 22, 12] for more details).

\section{GAME THEORY APPLICATIONS IN MICRO-GRID SYSTEMS}

In this section, a number of applications of game theory concepts in the field of micro-grids are covered. The power of game-theory approaches in solving such optimization problems is illustrated. Finally, some future opportunities for each approach are proposed.

\subsection{Cooperative games' applications}

\subsubsection{Power loss reduction in connected mode micro grid systems.}

In the existing power system, the main grid takes responsibility for meeting the power needs of all consumers in the electric system. The power is transmitted from the generating power plants to distribution communities over high voltage transmission lines, and then the power is transmitted from the distribution substations to the consumption areas (residential/industrial) over medium voltage transmission lines. Finally, the voltage is lowered to a low level voltage to be consistent with the voltage of the loads. This process of power transmission from the generation power plants down to the consumers is described to be centralized and is expected to have a lot of drawbacks as: 1) The power losses over the transmission lines are high due to the long distance between the generation and the consumption of the power in addition to the part of power lost in the transformer stage (lowering the high voltage to medium voltage and lowering the medium voltage to low voltage). 2) Any electrical fault will affect a wide area of the network. 3) The main grid is the only controller of the price of power and there is no chance for two-way power exchange to take place. 4) When Blackout occurs, it is expected to affect a massive part of the network if not the entire network.

In this regard, and due to its regional nature, the micro grid system is believed to solve the most of these problems. Micro grids can cooperate among themselves to locally exchange their power. This method is expected to reduce the load on the main grid, protect themselves against blackouts, and reduce the power losses on the transmission lines due to the short distance between the cooperative $\mu \mathrm{G}$ s.

In [23], a coalition formation game is constructed. The players of this game are a number of micro grids. Each $\mu \mathrm{G}$ has its own power capacity "amount of generated power" and each $\mu \mathrm{G}$ is responsible for feeding a group of loads with electric power. The authors of [23] seek to optimize "minimize" the cost of operation by minimizing the power losses. The authors assume that the system operates as a connected mode $\mu \mathrm{G}$ system. The system consists of a single Macro-Station in the center of the distribution network. This Macro-Station is connected from one side to the main grid through a power transformer and from the other side to a group of $\mu \mathrm{Gs}$. Each $\mu \mathrm{G}$ is considered as a local energy system which consists of local renewable energy resources that should have the ability to provide the local consumers with their needs of power. As the renewable energy sources' level of generation varies from hour to another hour, the net power of each $\mu \mathrm{G}$ is also changed due to the equation $\mathrm{P}_{\mathrm{i}}=\mathrm{P}_{\mathrm{Gi}}-\mathrm{P}_{\mathrm{Di}}$ where $\mathrm{P}_{\mathrm{Gi}}$ is the amount of power generated by the energy resources within $\mu G_{i}, P_{D i}$ is the amount of power consumed by the loads within $M G_{i}$. Hence, the net power $P_{i}$ can be formulated as follows:

$$
P_{i}= \begin{cases}\text { positive value } & \text { when } P_{G i}>P_{D i} \\ \text { negative value } & \text { when } P_{G i}<P_{D i} \\ \text { zero } & \text { when } P_{G i}=P_{D i}\end{cases}
$$

In the first two cases, The $\mu \mathrm{G}$ either has a power greater than its needs or less than its needs. Therefore, in both cases, the $\mu \mathrm{G}$ should trade this power (import or export it respectively) with the main utility grid or with nearby micro grids.

If the $\mu \mathrm{G}$ traded the power with the main grid, then the amount of power losses between $\mu \mathrm{G}_{\mathrm{i}}$ and the macro station is calculated using the equation: $\mathrm{P}_{\text {losses }}=\mathrm{I}^{2} \mathrm{R}+\alpha \mathrm{P}_{\text {flowing }}$ such that $\mathrm{I}$ is the current flowing through the transmission lines when the electrical energy is transmitted between the $\mu G_{i}$ and the macro station, $\mathrm{R}$ is the resistance of the transmission lines between $\mu G_{i}$ and the macro station, $\alpha$ is the amount of power dissipated in the transformer and $\mathrm{P}_{\text {flowing }}$ is the amount of power flowing between $\mu G_{i}$ and the macro station.

The non-cooperative utility function is: $\mathrm{u}(\{\mathrm{i}\})=-\mathrm{w}_{\mathrm{i}} \mathrm{p}_{\mathrm{i} 0}^{\text {loss }}$ where $\mathrm{w}_{\mathrm{i}}$ is the price paid by the $\mathrm{MG}_{\mathrm{i}}$ for each kilowatt of the power lost in the transmission phase and $\mathrm{p}_{\mathrm{i} 0}^{\text {loss }}$ is the amount of power dissipated in the transmission lines and in the transformer stage when the power is transmitted between the $\mu \mathrm{G}_{\mathrm{i}}$ and the main grid.

Exchanging the power locally with nearby micro grids and without depending on the utility grid is considered to be a general tendency as this cooperative manner decreases the power losses due to two reasons: 1) The short distance between the $\mu \mathrm{Gs}$ compared with the distance between the $\mu \mathrm{Gs}$ and the macro station. 2) There are no transformers between the $\mu \mathrm{Gs}$ so that the power dissipated in the macro station transformer is avoided.

The authors use a coalition formation game to group the $\mu \mathrm{G} s$ according to their needs in disjoint groups. Within each group, there are a number of power exporters and power importers and the power is coordinated within each $\mu \mathrm{G}$ to optimize "minimize" the power losses across the transmission lines. The utility gained by each coalition is then distributed over the members of the coalition using fair division method. $\mu \mathrm{G}$

The merge/split algorithm used to form the coalitions in [23] is described to be an NP-hard algorithm. This reason makes it not scalable and difficult to be used in real life applications. In [26], a hierarchical clustering algorithm is proposed to form coalitions among $\mu$ Gs. The complexity of this algorithm is quadratic which makes it scalable and more efficient to be used with large number of $\mu$ Gs. The authors of [26] describe the utility function of the non-cooperative case for certain $\mu G_{i}$ as:

$$
\mathrm{U}_{\mathrm{i}}=\frac{1}{1+\left|\mathrm{P}_{\mathrm{D}}-\mathrm{P}_{\mathrm{G}}\right|}
$$

From this formula, it is obvious that the less the power traded with the main grid, the more the benefits received by $\mu G_{i}$.

In the case of cooperative scheme, for a coalition $\mathrm{C} \subseteq \mathrm{N}$, the total energy transmitted within $\mathrm{C}$ can be calculated as 
$\mathrm{E}_{\mathrm{C}}=\sum_{\mathrm{i} \in \mathrm{C}}\left|\mathrm{P}_{\mathrm{D}}-\mathrm{P}_{\mathrm{G}}\right|$ and the overall losses between each two distinct $\mu$ Gs $(i, j)$ is $L_{C}=\sum_{i, j \in C} p_{\text {losses }}(i, j)$. Therefore, the utility of the coalition $\mathrm{C}$ can be calculated as:

$$
\mathrm{U}_{\mathrm{C}}=\frac{1}{1+\mathrm{E}_{\mathrm{C}}+\mathrm{L}_{\mathrm{C}}}
$$

To locate the payoff among the members of the coalition, the authors use the Shapley value division. For a coalitional game $(\mathcal{N}, \mathcal{V})$, the payoff of player $\mathrm{i}$ is calculated using the formula:

$$
\varphi_{\mathrm{i}}(\mathcal{N}, \mathcal{V})=\sum_{\mathrm{s} \subseteq \mathrm{N}-\{\mathrm{i}\}} \frac{|\mathrm{S}| !(|\mathrm{N}|-|\mathrm{S}|-1) !}{|\mathrm{N}| !}
$$

Where $\mathrm{S} \subseteq \mathrm{N}$ is a coalition of $\mu \mathrm{Gs}$ and $\mathrm{N}$ is the total number of players " $\mu \mathrm{Gs}$ " included in the game.

A pricing scheme is used to study the effect of pricing on the coalition formation process. The results show that the HR clustering algorithm used is less complex, faster and more scalable than the merge/split algorithm. Therefore, it is more suitable to be used with large number of $\mu \mathrm{Gs}$.

The work in [23] is carried out on a small size $\mu \mathrm{G}$ system. The authors assume that the network studied is established on a $10 \mathrm{~km} \times 10 \mathrm{~km}$ area, but this area is too small to study the effect of the algorithm on it. The authors of [25] study the same problem but on a more realistic network with a size of $100 \mathrm{~km} \times 100 \mathrm{~km}$. Two dynamic learning processes are proposed to construct a coalition structure and to achieve the highest power saving. The coalitions formed are not necessarily to be mutually exclusive and coalitions with only one $\mu \mathrm{G}$ "singletons" are permitted. For coalitions with more than one $\mu \mathrm{G}$, one $\mu \mathrm{G}$ with excess power is connected to several $\mu \mathrm{Gs}$ that face lack of power challenges. In contrary, for singleton coalitions, the $\mu \mathrm{G}$ only exchanges the power with the main grid. The work in [25] uses the parameters of the $\pi$-model of medium length transmission line to express the formula of the power losses as follows:

$$
P_{\text {loss }}=P_{s}-P_{r}=V_{s} \cdot I_{s} \cdot \cos \left(\theta_{s}\right)-V_{r} \cdot I_{r} \cdot \cos \left(\theta_{r}\right)
$$

where $P_{S}$ is the sent power, $P_{r}$ is the received power, $V_{\mathrm{s}}, \mathrm{I}_{\mathrm{s}}, \cos \left(\theta_{\mathrm{s}}\right)$ are the sending end voltage, current and power factor, respectively, and $V_{r}, I_{r}, \cos \left(\theta_{r}\right)$ are the receiving end voltage, current and power factor, respectively. The results of the work of [25] show that the dynamic learning technique used in the coalition formation process converges to a stable state. In addition, the results show that the power losses in the case of cooperation represent only $10 \%$ of the losses in the case of non-cooperative case.

The authors of [30] pay more attention to improve the reliability and efficiency of the entire system rather than individual $\mu$ Gs. The work done in $[23,25,26]$ assumes that the sale price of power is the same for all $\mu \mathrm{Gs}$, the models created also study the system as a totally connected network i.e. there is an electric transmission line between any two $\mu \mathrm{Gs}$. This assumption causes the system to be more complicated, increases the opportunities of electrical faults and decreases the effectiveness of the proposed algorithm, from an economical point of view.

The work in [30] proposes a multi-stage algorithm. The presented algorithm consists of three steps:

Step \#1: Auction Theory is used to match the sellers ( $\mu \mathrm{G}$ with surplus power) to buyers ( $\mu \mathrm{Gs}$ with lack of power) in order to calculate the maximum utility of each possible coalition. The utilization of Auction Theory reflects the incentive of each $\mu \mathrm{G}$ and helps in increasing the stability of each coalition.

Step \#2: the payoff is allocated among the members of each coalition using the Shapley value solution concept.

Step \#3: merge and split algorithm is introduced to reach the optimal coalition formation partitions.

The algorithm used considers reasonable connections between $\mu \mathrm{Gs}$. This consideration decreases the time complexity of the algorithm and increases its scalability.

The results show that the proposed algorithm can help in increasing not only the individual payoff of each $\mu \mathrm{G}$, but also the utility of the overall network.

Future opportunities for power loss reduction in $\mu \mathrm{G}$ systems:

- The model could be developed to study the effect of cooperation on the economic load dispatch including losses in thermal plants.

- The utility function could be multi-objective to capture not only power losses but also other parameters such as the voltage drop across the transmission lines.

- As the power losses are always proportional to the square of the current flowing through the transmission lines, quadratic programming should be used to coordinate the power transfer within every coalition.

- The solutions of cooperative games such as the nucleus and Shapley value should be used instead of fair division in [23] in order to increase the stability of the system as the stability is very important in electric systems.

- Meta-heuristic algorithms for coalition formation could be used to decrease the time of processing and to increase the scalability of the model.

- Bargaining techniques could be used to study the conditions under which coalitions will be constructed.

- More future opportunities can be found in [5].

\subsubsection{Discomfort level minimizing in isolated mode micro-grid systems.}

The $\mu \mathrm{G}$ system can operate in two different modes: 1) The connected mode at which the $\mu \mathrm{G}$ is connected to the main grid and can exchange the power with it. 2) The islanded mode at which the $\mu \mathrm{G}$ operates in an autonomous way to cover the needs of its loads.

The work in [24] studies the effect of the absence of the maingrid on the hardship faced by the $\mu$ Gs to fulfill all the needs of their connected loads. Agent-Based dynamic coalition formation algorithm is proposed. The aim of cooperation is to decrease the overall discomfort of the system.

When the $\mu \mathrm{G}$ is isolated from the main grid, it can neither sell energy to nor buy energy from the main grid. Therefore, at a certain time slot $i$ and due to its intermittent generation nature, a certain $\mu \mathrm{G}$ can suffer from power shortage. This power shortage could be measured by the notion of "power deficit". The deficit level takes a value between 0 and 10 and can be calculated as: 


$$
\mathrm{P}_{\operatorname{Def}(\mathrm{i})}=\operatorname{Max}\left[\frac{\mathrm{P}_{\mathrm{D}(\mathrm{i})}-\mathrm{P}_{\mathrm{G}(\mathrm{i})}}{\mathrm{P}_{\mathrm{D}(\mathrm{i})}}, 0\right] * \text { maxrange }
$$

such that: $\mathrm{P}_{\operatorname{Def}(\mathrm{i})}$ is the level of power deficit at time slot $i$, $\mathrm{P}_{\mathrm{D}(\mathrm{i})}, \mathrm{P}_{\mathrm{G}(\mathrm{i})}$ are the load and generated power amount at time slot $i$, respectively. maxrange is the maximum value of deficit which is defined to be 10 .

The level of deficit takes a value of 0 if the generated amount of power is greater than or equal to the loads and it takes a value of 10 if the generated power is 0 . The levels of deficit can be concluded as:

$$
\mathrm{P}_{\operatorname{Def}(\mathrm{i})}= \begin{cases}0 & \text { when } \mathrm{P}_{\mathrm{G}(\mathrm{i})} \geq \mathrm{P}_{\mathrm{D}(\mathrm{i})}, \mathrm{P}_{\mathrm{G}(\mathrm{i}) \neq 0} \\ 10 & \text { when } \mathrm{P}_{\mathrm{G}(\mathrm{i})}=0, \mathrm{P}_{\mathrm{D}(\mathrm{i})} \neq 0 \\ 0<x<10 & \text { when } \mathrm{P}_{\mathrm{D}(\mathrm{i})} \geq \mathrm{P}_{\mathrm{G}(\mathrm{i})}, \mathrm{P}_{\mathrm{G}(\mathrm{i}) \neq 0}\end{cases}
$$

The level of discomfort "LOD" is assumed to be a continuous polynomial function which takes the power deficit " $P_{\text {Def(i) }}$ " as an input and the output of it varies between 0 and 10 where 0 means that the $\mu \mathrm{G}$ is totally comfort and 10 means that the $\mu \mathrm{G}$ is totally discomfort. LOD can be calculated by the equation:

$$
\mathrm{LOD}=\mathrm{aP}_{\operatorname{Def}(\mathrm{i})}+\mathrm{b}\left(\mathrm{P}_{\operatorname{Def}(\mathrm{i})}\right)^{2}+\mathrm{c}\left(\mathrm{P}_{\operatorname{Def}(\mathrm{i})}\right)^{3}
$$

such that $(\mathrm{a}, \mathrm{b}, \mathrm{c})^{\mathrm{T}}=(0.1,-0.01,0.01)^{\mathrm{T}}$.

The authors propose a coalition formation game at which the $\mu$ Gs with high level of discomfort can alleviate the load from their shoulders and decrease the level of discomfort by getting power from other $\mu \mathrm{Gs}$ with low level of discomfort. The proposed algorithm significantly reduces the overall level of discomfort of the entire network by decreasing the aggregate level of discomfort of each coalition. The aggregate level of discomfort of each coalition $\mathrm{C} \subseteq \mathrm{N}$ can be calculated as:

$$
\mathcal{V}\left(\mathrm{C}_{\mathrm{j}}\right)=\sum_{\mathrm{i}=1}^{|\mathrm{S}|} \operatorname{LOD}_{\mathrm{i}}
$$

where $\mathrm{j}$ is the coalition number, and $|\mathrm{S}|$ is the number of $\mu \mathrm{Gs}$ concluded in the $\mu \mathrm{G}$ number $\mathrm{j}$.

\section{Future opportunities for LOD reduction in isolated mode $\mu \mathrm{G}$ systems:}

- Goal programming could be used to make a balance between the level of autonomy of the $\mu \mathrm{Gs}$ and the level of stability of coalitions.

- Study the effect of using storing devices on the process of power exchange.

- Non-cooperative game theory approaches such as auction games could be used to study the situation.

- Propose a hybrid multistage algorithm that contains both cooperative and forecasting techniques to reduce the LOD.

\subsubsection{Cost reduction in $\mu G$ distribution networks using a coalition formation game.}

The authors of [29] propose a coalition formation algorithm that captures not only the power losses but also the cost of the purchased power. The proposed algorithm is described to be a general algorithm as it involves two phases. Phase $\mathrm{I}$ is dedicated to form coalitions in the case of connected mode. In contrary, phase II is concerned with the optimization of coalitions in the case of islanded mode.

The mode of operation is determined according to two conditions:
Condition no.1: $\mathrm{P}_{\mathrm{i}}=\mathrm{P}_{\mathrm{Gi}}-\mathrm{P}_{\mathrm{Di}}>\Delta \mathrm{q}_{\mathrm{i}}$ and $\Delta \mathrm{q}_{\mathrm{i}}>\max \mathrm{P}_{\mathrm{i}}^{\text {loss }}$ form macro station $\mathrm{i} \in \mathrm{N}$ where $\mathrm{N}$ is the set of macro stations exists in the network, $\Delta q_{i}$ is the threshold of mode transfer and $\max \mathrm{P}_{i}^{\text {loss }}$ is the maximum amount of power lost by macro station i during power transfer. The power losses could be calculated using the equation of $\mathrm{P}_{\text {losses }}$ aforementioned in [23].

Condition no.2: $\mathrm{P}_{\mathrm{i}}=\mathrm{P}_{\mathrm{Gi}}-\mathrm{P}_{\mathrm{Di}}<\max \mathrm{P}_{\mathrm{i}}^{\text {loss }}$ or $\mathrm{R}_{\mathrm{ij}}=\infty$ which means that the macro station i excess power can't cover the power exchange losses. Therefore, the islanded mode is recommended because it is more economical at this case.

At the start of operation, the macro station (MS) tests the network to determine its mode of operation. If condition no.1 is satisfied, then the connected mode will be activated and if condition no. 2 is satisfied, then the islanded mode is activated. Therefore, the MS periodically checks the conditions to choose the appropriate mode of operation.

In the case of connected mode, the power could be exchanged between the main utility and the macro stations. The authors assume that the price of power varies from time interval to another and from a certain utility to another one. If the amount of power exchanged is $\mathrm{q}$, then the prices could be expressed as:

$$
\mathrm{C}_{\text {on-peak }}(\mathrm{q})>\mathrm{C}_{\text {off-peak }}(\mathrm{q})>\mathrm{C}_{\text {cop }}^{\mathrm{i}}(\mathrm{q})>\mathrm{C}_{\text {noncop }}^{\mathrm{i}}(\mathrm{q})
$$

where $\mathrm{C}_{\text {on-peak }}(\mathrm{q})$ is the price of amount $\mathrm{q}$ of power purchased from the main utility at peak time, $\mathrm{C}_{\text {off-peak }}(\mathrm{q})$ is the price paid for the same amount of power purchased from the main utility through the day at off-peak times, $\mathrm{C}_{\text {noncop }}^{\mathrm{i}}(\mathrm{q})$ is the worth of $\mathrm{C}_{\text {noncop }}^{\mathrm{i}}(\mathrm{q})$ transferred from $\mathrm{MS}_{\mathrm{i}}$ to the main grid and $\mathrm{C}_{\text {cop }}^{\mathrm{i}}(\mathrm{q})$ is the worth of amount $\mathrm{q}$ of power traded locally to other MSs within the coalition. The authors also take in consideration the fee paid by the MSs to rent the transmission lines to transfer power among themselves. The renting fee between MS i and MS j is denoted by $\mho_{i, j}=$ $\alpha \mathrm{d}_{\mathrm{i}, \mathrm{j}} \forall \mathrm{i}, \mathrm{j} \in \mathrm{N}$ such that $\alpha$ is the rate of renting the transmission lines and $\mathrm{d}_{\mathrm{i}, \mathrm{j}}$ is the distance between $\mathrm{MS}_{\mathrm{i}}$ and $\mathrm{MS}_{\mathrm{j}}$. The objective of coalition at this case is to minimize the amount of money paid for purchasing power in addition to reducing the power dissipation during exchanging power. The objective function could be expressed as:

$$
\min \mathrm{w}_{1} \sum \mathrm{P}_{\text {loss }}+\mathrm{w}_{2} \sum \mathrm{P}_{\text {cost }}
$$

where $\sum \mathrm{P}_{\text {loss }}$ denotes the total power dissipation, $\sum \mathrm{P}_{\text {cost }}$ is the total cost for purchased amount of power and $\mathrm{w}_{1}, \mathrm{w}_{2}$ are constants used as goal programming weights and the value of them depends on the priority of the MS is to minimize the power dissipation or to decrease the costs of power purchased.

In the case of islanded mode, the MSs are isolated from the main grid. Therefore, the power is distributed among themselves in a way to decrease the payment for the purchased power. Hence, the objective function is:

$$
\min \sum \mathrm{P}_{\operatorname{cost}}(\mathrm{t})
$$

where $\sum P_{\text {cost }}(t)=\sum \tau_{u}\left(q_{u}\right)$ and $\tau_{u}$ is the price for unit power from $\mu \mathrm{G}_{\mathrm{u}}$ and $\mathrm{q}_{\mathrm{u}}$ is the amount of power transferred from or to $\mu \mathrm{G}_{\mathrm{u}}$.

The results show that the proposed algorithm significantly decreases the cost of power in both connected and islanded mode. 
Future opportunities for cost reduction in $\mu \mathrm{G}$ distribution networks:

- Study the effect of dynamic pricing on the process of forming the cooperative groups.

- Study the process of optimal coalition formation besides the optimization problem.

- Calculate the payoff of each MS and $\mu \mathrm{G}$ as the power of game theory is to allocate the individual payoffs of each player to guarantee the stability of the coalitions.

- The algorithm could be updated to be agent based.

- The algorithm needs to be more scalable by capturing wide number of MSs and $\mu$ Gs to be more realistic.

- Apply the algorithm in real life to measure its effect in a practical way.

\subsubsection{Cooperative game theoretic model for load managing and scheduling in residential community $\mu G$ systems.}

The technology of smart meters "SMs" has significant contributions in the improvement of smart grid systems. This technology besides DGs, help in transforming the residential end users from passive consumers of power to active prosumers i.e. producers and consumers of electrical energy. At the residential distribution level, and using the technologies of DGs and SMs, residential homes could be described to be smart. Each smart home contains main components such as: 1) Renewable energy resources like solar cells and micro wind turbines. 2) Smart meters that help in the construction of bi-directional communication layer between the smart homes and the main utility in addition to providing the home with important information like level of energy consumption and the instantaneous price of power. 3) Load scheduling unit which is responsible for scheduling the loads in a way to minimize the cost the purchased power by shifting loads away from the peak load times. 4) Energy management centers which is responsible for the process of exchanging power. 5) Energy storage devices to store the power when it exceeds the needs of the loads, and finally 6) the home appliances which represent the loads of the home. Each smart home represents a node in the micro grid network and connected to the main utility via the energy management center.

The authors of [33] propose a model for a micro grid system based on residential community. The proposed model depends on the concepts of coalition formation games. The aim of the model is to exchange excessive power locally among the smart homes and externally with the utility grid. The players of the game are the smart homes which are classified into three kinds according to their construction to: 1) Homes with no DERs. 2) Homes with DERs but with no energy storage devices. 3) Homes with DERs and with energy storage devices. The authors present two designs for the process of cooperation among residential communities. In the first design, the cooperation process is decentralized and the agents of homes can negotiate with each other directly without any mediator. On the contrary, the homes in the second design send their surplus power to the smart community manager which decides the amount and the price of power to be purchased to each home and to the main utility. The authors conclude that the cooperative model optimally schedules the loads, reduce the cost of purchasing power, reduce the power losses and the harmful emissions, and relieve the loads of the main utility grid.

Future opportunities for load managing and scheduling in residential community $\mu \mathrm{G}$ systems using cooperative game concepts:

- The solution concepts such as Shapley value and the Nucleus should be used to allocate the payoff "the gains" among the homes.

- Study the non-cooperative behavior of homes equipped by storage devices.

- Use the concepts of bargaining to study the conditions under which cooperation will take place.

- Stability of coalitions should be studied to assure that no player has an intensive to leave his coalition.

\subsection{Non-cooperative games applications}

\subsubsection{Bi-level non-cooperative game for optimal contract prices allocation.}

Small-scale distributed generators (DGs) are counted as one of the corner stones of Micro Grid technology. Each DG has a cost of generation due to its initial costs, operation and maintenance costs. This cost varies from one DG to another depending on the geographical location, weather and the method of power generation. Therefore, the rate of unit power differs from one DG to another. This variation in the cost of power leads to a competitive behavior between the DGs owners in a way to maximize their sales income.

In [27], a non-cooperative game is utilized to find the optimal contract price of electric power. The players of this game are the owners of the DGs, the strategies of the players are the prices they offer for selling their generated amount of electric power. The authors propose a bi-level non-cooperative static game similar to the Stackelberg game. The game involves two agents. The first agent "the leader" represents the authors of the DGs and the second agent "the follower" represents the distribution companies "DisCos". The game consists of two stages. At the first stage, the owners of the DGs make their offer aiming to maximize their payoff. The payoff of the owners of the DGs is represented as:

$$
\mathrm{f}=\max \left(\mathrm{C}_{\text {inc }}-\mathrm{C}_{\text {init }}-\mathrm{C}_{\text {oper\& main }}\right)
$$

where $\mathrm{C}_{\text {inc }}$ is the income of the DGs and $\mathrm{C}_{\text {init }}, \mathrm{C}_{\text {oper\& main }}$ are the initial and operating and maintenance costs, respectively. At the second stage, the DisCos select the more efficient offer in order to minimize their payment for purchasing power. The payoff of the DisCos is represented as:

$$
\mathrm{G}=\min \left(\mathrm{C}_{\text {sub }}+\mathrm{C}_{\mathrm{inc}}\right)
$$

where $\mathrm{C}_{\text {sub }}$ is the cost of the amount of power purchased from a certain substation. The payoff gained by each DG owner depends not only on the price offered by him, but also on the price offered by other DGs owners and on the marginal price of electricity market. Therefore, the game theory is adopted to find the Nash-equilibrium point i.e. the point at which the optimal contract price is obtained.

The others outline the steps of the game as follows:

Step \#1: The DGs owners offer their prices.

Step \#2: the payoff of the owner of the distributed generator $D G_{i}$ is denoted by $\pi_{i}$ and is calculated using the payoff function " $\mathrm{f}$ " mentioned above. 
Step \#3: the NE point is calculated by:

$$
\pi_{\mathrm{i}}\left(\mathrm{s}_{\mathrm{i}}^{*}, \mathrm{~s}_{\mathrm{N}-\{\mathrm{i}\}}^{*}\right) \geq \pi_{\mathrm{i}}\left(\mathrm{s}_{\mathrm{i}}, \mathrm{s}_{\mathrm{N}-\{\mathrm{i}\}}^{*}\right) \forall \mathrm{i} \in \mathrm{N}, \forall \mathrm{s}_{\mathrm{i}} \in \mathrm{S}_{\mathrm{i}}
$$

Where $s_{i}^{*}$ is the NE vector.

Step \#4: the payoff of the DisCos is calculated using the payoff function "G" mentioned above.

The results show that by increasing the capacity of the DGs, the amount paid for the purchased power is decreased and the also the payment of DGs for their power sale is increased.

In addition to the contract price offers, the authors of [28] also investigate the effect of the location of the DG units on the profit it can gain and the impact of the dispatch of the DG unit on the performance of the entire network. For example: if a certain DG dispatching harms the power flow in the network by increasing the power losses or causing the voltage to be out of the appropriate range; then, such DG is rejected, even if the contract price offer of it is lower than other DGs offers or lower than the whole market price.

The results of [28] show that DGs located in central buses have the ability to increase their profits more than those DGs located in non-central buses. Therefore, the competition among the DGs owners depends not only on the price offered for the generated power, but also on the location of the DG unit in the network. The results also illustrate that the equilibrium contract prices can be reduced by increasing the size and the number of the DG units in the network.

\section{Future opportunities for optimal contract prices} allocation:

- Dynamic auction could be used to study the situation instead of the one shot game proposed.

- Non-cooperative game between the $\mu \mathrm{Gs}$ owners and the DisCos could be adopted in order to optimize the gains of the DGs owners and to match the power needs of the DisCos.

- Study the effect of the storage devices on the behavior of the players.

- Cournot model and Bertrand model could be used to analyze the performance of the power market.

\subsubsection{Distribution level energy market model using non-cooperative game theory.}

In contrast to the recent power system, the energy in smart grids is described to be bidirectional (the consumers in the distribution level can construct their own $\mu$ Gs depending on photovoltaic cells, micro wind turbines, and/or diesel generators). Therefore, they can trade power with other consumers and/or with the main utility. This behavior of consumers encourages the researchers to study the energy trading market among these distribution level micro grids "DL $\mu \mathrm{Gs}$ " and the utility and retails "UTRs".

The authors of [31] propose a market model based on multiplayer non-cooperative game theory to study the effect of this approach of power trading on the payoff of each DL $\mu \mathrm{G}$ and on the overall utility of the main grid. The presented model captures the reputation of each player which makes it scalable and affords autonomy. The market is constructed to encompass both DL $\mu \mathrm{Gs}$ and the UTRs. The generation levels of DL $\mu \mathrm{Gs}$ vary with time during the day as they depend mainly on renewable energy sources. Therefore, when the generated power is more than the loads, DL $\mu$ Gs participate in the market as sellers, otherwise they participate as buyers. Forecasting techniques are essential for the model as DL $\mu$ Gs should have the ability to predict the amount of generation and loads in each hour during the day.

The objective of the work is to maximize the local power generation in addition to reducing the costs of power production. The utility function for the $i^{\text {th }} D L \mu G$ at time $t$ could be written in the form:

$$
\mathrm{U}_{\mathrm{t}}^{\mathrm{DL} \mu \mathrm{G}, \mathrm{i}}=\mathrm{R}_{\mathrm{t}}^{\mathrm{DL} \mu \mathrm{G}, \mathrm{i}}-\mathrm{C}_{\mathrm{t}}^{\mathrm{DL} \mu \mathrm{G}, \mathrm{i}}
$$

where $R_{t}^{D L \mu G, i}$ is the revenue function of the ith DL $\mu G$ at time $t$ and $C_{t}^{D L \mu G, i}$ is the cost function of the $i^{\text {th }} D L \mu G$ at time $t$. Where the utility function of the $\mathrm{k}^{\text {th }}$ RTU could be expressed as:

$$
\mathrm{U}_{\mathrm{t}}^{\mathrm{UTR}, \mathrm{k}}=\alpha_{\mathrm{sell}, \mathrm{t}}^{\mathrm{UTR}, \mathrm{k}} * \mathrm{P}_{\mathrm{sell}, \mathrm{t}}^{\mathrm{UTR}, \mathrm{k}}+\mathrm{s}_{\mathrm{t}}^{\mathrm{k}}
$$

where $\alpha_{\text {sell,t }}^{\mathrm{UTR}, \mathrm{k}}$ represents the rate of unit power sold by the kth RTU at time $t, \mathrm{P}_{\text {sell,t }}^{\mathrm{UTR}, \mathrm{k}}$ is the amount of power sold by the kth RTU at time $t$ and $s_{t}^{k}$ is the service fee charged by each DL $\mu \mathrm{G}$ using the services of the kth RTU.

The results show that the utilization of the model and the extreme seeking algorithm for calculating Nash equilibrium causes the individual payoff of each DL $\mu \mathrm{G}$ increases in comparison with the case of the nonexistence of such market model, the overall utility of the UTRs is reduced due to the dependence on local power trading, and the market cleaned price is also reduced due to the existence of cheaper generation sources.

Future opportunities for Distribution level energy market model using non-cooperative game theory:

- Storage devices presence effect on the market should be studied.

- Bidirectional power exchange between the DL $\mu$ Gs and the UTRs should be studied as in sometimes the surplus power should be exchanged with the utility when there is a surplus power than the needs all DL $\mu \mathrm{Gs}$.

- Stackelberg game model could be used to study the system in a bi-level manner using the UTRs as a leader and the DL $\mu \mathrm{Gs}$ as a follower.

\subsubsection{Non-cooperative game theoretic approach for reactive power and voltage control in power system networks.}

Power quality is an essential term in power system networks, especially in micro-grid systems. It is used to describe the properties of the waveform of the transmitted power in the electrical network. The voltage is one of the factors that greatly affect the evaluation of power quality in the system. To guarantee a good quality of power, the voltage should be maintained within a prescribed range, and to ensure the stability of voltage level, reactive voltage controllers such as VAR compensators, shunt reactors and overexcited synchronous generators should be periodically dispatched. The injection or dispatching of reactive power could increase the power losses and the loading of the system if it is not properly controlled. Due to the different natures of the voltage-control devices, the interaction between these devices is too complicated. Thus, the control of such devices is too difficult. 
The authors of [32] use the concepts of game theory to simplify the interaction between such devices in a way to ensure that the voltage is within the acceptable range during the dispatching of reactive power sources and compensators. The objective of the proposed approach is to reduce the injection of reactive power during the activities of voltage control in addition to ensuring that the voltage is within the acceptable range. The players involved in the game are the devices used to control the reactive power such as shunt reactors, static VAR compensators and on load tap changers "OLTCs". The payoff of each player is determined by the amount of reactive power injected by the device. The authors provide two game theoretic approaches. Both of them are modeled as a non-cooperative extensive form game.

In the first game, the sequence of the players' actions is of order: generator - OLTC - compensator. This game illustrates that the reactive power injection is divided among the compensators. Therefore, the demand of reactive power from the generators is decreased.

On the contrary, in the second game, the reactive power injection is shared among the generators alleviating the demand of the reactive power from the compensators.

The results show that the utilization of the game theoretic approach reduces the reactive power injection in addition to decreasing the power losses of the entire system.

\section{Future opportunities for reactive power and voltage} control in power system networks:

- The model should be developed to be more scalable to capture a large number of nodes.

- The model could be improved to be hybrid to capture the effect of cooperation between different players and its effect on the voltage and the reactive power in addition to the non-cooperative approach studied.

- The equilibrium should be studied to find the equilibrium point.

\subsubsection{Locating and sizing distributed generators in distribution networks using Nash equilibrium.}

Distribution generation technology is believed to have a great effect on the improvement of the distribution networks in the system of smart grids. DGs are connected to the network at low voltage levels i.e. in the distribution network near to consumers. The injection of DGs in the distribution level should be at certain locations and with prescribed capacities to enhance the stability of the electric power in the network. Therefore, various electrical quantities need to be optimized to guarantee the goodness of the system during the phase of network design. The dispatching of DGs should be optimized to reduce the costs of operation, decrease the power losses and to fix the voltage within the accepted ranges.

The authors of [34] provide a novel method based on noncooperative game theory to find optimal locations and capacities of DGs in order to reduce the overall power losses in addition to enhancing the voltage profile of the network. The system is modeled as a 2-person non-cooperative game. The first player represents a group of DGs seeking to reduce the overall power losses of the grid. Thus, the objective of the first player is to minimize the power losses of each feeder. The total losses at feeder number I could be formulated as:

$$
\mathrm{P}_{\mathrm{i}, \text { loss }}=\sum_{\mathrm{k}=1}^{\mathrm{n}} \mathrm{P}_{\text {loss }}(\mathrm{k}, \mathrm{k}+1)+\sum_{\mathrm{k}=1}^{\mathrm{n}} \mathrm{Q}_{\text {loss }}(\mathrm{k}, \mathrm{k}+1)
$$

where $P_{\text {loss }}(k, k+1)=R_{k} \times \frac{P_{k}^{2}+Q_{k}^{2}}{v_{k}^{2}}$ and $Q_{\text {loss }}(k, k+1)=$ $X_{k} \times \frac{P_{k}^{2}+Q_{k}^{2}}{v_{k}^{2}}$ represent the active and reactive power losses between buses $k$ and $k+1$, respectively. Such that $R_{k}$ and $X_{k}$ are the resistance and reactance between buses $\mathrm{k}$ and $\mathrm{k}+1$, respectively and $P_{k}, Q_{k}$ represent the amounts of active and reactive power flows out from the bus $\mathrm{k}$. The second player represents a group of DGs seeking to improve the voltage to be within the acceptable range. Therefore, the objective of the second player is to guarantee that the per-unit value of the voltage is greater than 0.94 PU and less than 1.06 PU. Each player can make one of the following actions to maximize his payoff: 1) Change the sizes of DGs and leave the locations unchangeable. 2) Change the locations of the DGs leaving the sizes unchangeable. 3) Change both of the capacities and locations of the DGs.

The authors utilize a method depending on genetic algorithm to find the point of Nash equilibrium. The GA Nash algorithm proposed is faster in finding the Nash point of equilibrium. The results show that the bus voltages are improved and the overall losses of the network are reduced.

\section{Future opportunities for locating and sizing DGs in the} distribution networks:

- Increase the number of DGs used in the system to be more realistic.

- Bi-level games such as Stackelberg game could be used to study the system in a dynamic way.

- Goal programming could be used to find an optimal point due to the preference of the network designer.

\section{CONCLUSION}

In this paper, the importance of micro grid technology in smart grid systems has been explained. The article presented the technical problems facing micro grid technology, introduced the basics of game theory, summarized its branches and the importance of each branch, provided a comprehensive overview of the applications of game theory in the systems of micro grids and distribution level networks, and presented some future opportunities that could be used as keys to future researches. The surveyed work shows the power of game theory as a promising decision making tool in treating a lot of technical problems facing the utilization of micro grids. The focus in this article was mainly on the applications of game theory in solving the power exchange problems. However, game theory has a lot of applications in various aspects in smart grids such as the communication between $\mu \mathrm{Gs}$, the protection against various electrical faults and the process of managing and scheduling loads in the demand side.

\section{REFERENCES}

[1] Hartono, B.S. and Setiabudy, R., 2013, June. Review of microgrid technology. In QiR (Quality in Research), 2013 International Conference on (pp. 127-132). IEEE.

[2] Jiayi, H., Chuanwen, J. and Rong, X., 2008. A review on distributed energy resources and MicroGrid. Renewable and Sustainable Energy Reviews, 12(9), pp.2472-2483. 
[3] Hatziargyriou, N., 2008. Microgrids [guest editorial]. IEEE Power and Energy Magazine, 6(3), pp.26-29.

[4] Soshinskaya, M., Crijns-Graus, W.H., Guerrero, J.M. and Vasquez, J.C., 2014. Microgrids: Experiences, barriers and success factors. Renewable and Sustainable Energy Reviews, 40, pp.659-672.

[5] Saad, W., Han, Z., Poor, H.V. and Basar, T., 2012. Game-theoretic methods for the smart grid: An overview of microgrid systems, demand-side management, and smart grid communications. IEEE Signal Processing Magazine, 29(5), pp.86-105.

[6] Saad, W., Han, Z., Debbah, M., Hjørungnes, A., \& Basar, T. (2009). Coalitional game theory for communication networks: A tutorial. arXiv preprint arXiv:0905.4057.

[7] Carmichael, F. (2005). A guide to game theory. Pearson Education.

[8] Nash, J. (1951). Non-cooperative games. Annals of mathematics, 286-295.

[9] Nash, J. F. (1950). Equilibrium points in n-person games. Proceedings of the national academy of sciences, 36(1), 48-49.

[10] Myerson, R. B. (2013). Game theory. Harvard university press.

[11] Basar, T., \& Olsder, G. J. (1999). Dynamic noncooperative game theory (Vol. 23). Siam.

[12] Peleg, B., \& Sudhölter, P. (2007). Introduction to the theory of cooperative games (Vol. 34). Springer Science \& Business Media.

[13] Yeung, D. W., \& Petrosjan, L. A. (2006). Cooperative stochastic differential games. Springer Science \& Business Media.

[14] Branzei, R., Dimitrov, D., \& Tijs, S. (2008). Models in cooperative game theory (Vol. 556). Springer Science \& Business Media.

[15] Vincent, T. L., \& Brown, J. S. (2005). Evolutionary game theory, natural selection, and Darwinian dynamics. Cambridge University Press.

[16] Smith, J. M. (1982). Evolution and the Theory of Games. Cambridge university press.

[17] Mailath, G. J. (1998). Do people play Nash equilibrium? Lessons from evolutionary game theory. Journal of Economic Literature, 36(3), 1347-1374.

[18] Mei, S., Wei, W., \& Liu, F. (2017). On engineering game theory with its application in power systems. Control Theory and Technology, 15(1), 1-12.

[19] Chattopadhyay, S., \& Chatterjee, R. (2019). Understanding Auctions. Routledge.

[20] Kalai, E., \& Smorodinsky, M. (1975). Other solutions to Nash's bargaining problem. Econometrica, 43(3), 513518 .

[21] Kalai, E. (1977). Proportional solutions to bargaining situations: interpersonal utility comparisons. Econometrica: Journal of the Econometric Society, 1623-1630.
[22] Binmore, K., Rubinstein, A., \& Wolinsky, A. (1986). The Nash bargaining solution in economic modelling. The RAND Journal of Economics, 176-188.

[23] Saad, W., Han, Z., \& Poor, H. V. (2011, June). Coalitional game theory for cooperative micro-grid distribution networks. In 2011 IEEE international conference on communications workshops (ICC) (pp. 15). IEEE.

[24] Yasir, M., Purvis, M., Purvis, M., \& Savarimuthu, B. T. R. (2015, October). Dynamic coalition formation in energy micro-grids. In International Conference on Principles and Practice of Multi-Agent Systems (pp. 152168). Springer, Cham.

[25] Shams, F., \& Tribastone, M. (2015, September). Power Trading Coordination in Smart Grids Using Dynamic Learning and Coalitional Game Theory. In International Conference on Quantitative Evaluation of Systems (pp. 54-69). Springer, Cham.

[26] Chakraborty, S., Nakamura, S., \& Okabe, T. (2014, October). Scalable and optimal coalition formation of microgrids in a distribution system. In IEEE PES Innovative Smart Grid Technologies, Europe (pp. 1-6). IEEE.

[27] Moradi, M. H., Abedini, M., \& Hosseinian, S. M. (2015). A combination of evolutionary algorithm and game theory for optimal location and operation of DG from DG owner standpoints. IEEE Transactions on Smart Grid, 7(2), 608-616.

[28] López Lezama, J. M., Contreras, J., \& Padilha Feltrin, A (2012). Contract pricing evaluation of distributed generation: a game theory approach. Ingeniería y Desarrollo, 30(2), 182-198.

[29] Xu, Y., Hou, E., \& Ansari, N. (2014, December). Economic coalition strategies for cost reductions in microgrids distribution networks. In 2014 IEEE Global Communications Conference (pp. 2703-2708). IEEE.

[30] Mei, J., Chen, C., Wang, J., \& Kirtley, J. L. (2019). Coalitional game theory based local power exchange algorithm for networked microgrids. Applied energy, 239, 133-141.

[31] Bhatti, B. A., \& Broadwater, R. (2019). Energy trading in the distribution system using a non-model based game theoretic approach. Applied Energy, 253, 113532.

[32] Idehen, I., Abraham, S., \& Murphy, V. (2018, February). Reactive power and voltage control in a power grid: A game-theoretic approach. In 2018 IEEE Texas Power and Energy Conference (TPEC) (pp. 1-6). IEEE.

[33] Khaskheli, S., Halepoto, I. A., \& Khalid, A. (2019). RESIDENTIAL COMMUNITY MICRO GRID LOAD SCHEDULING AND MANAGEMENT SYSTEM USING COOPERATIVE GAME THEORY. $3 C$ Tecnologia

[34] Beiranvand, A., Abuhilaleh, M., \& Li, L. (2017, August). A novel method for optimizing distributed generation in distribution networks using the game theory. In 2017 20th International Conference on Electrical Machines and Systems (ICEMS) (pp. 1-5). IEEE. 